\title{
Mechanical properties of BBi compound under pressure
}

\author{
Salah Daoud \\ Laboratoire Matériaux et Systèmes Electroniques (LMSE), Université de Bordj Bou Arreridj, 34000, Algérie \\ E-mail: salah_daoud07@yahoo.fr
}

Copyright $\odot 2015$ Salah Daoud. This is an open access article distributed under the Creative Commons Attribution License, which permits unrestricted use, distribution, and reproduction in any medium, provided the original work is properly cited.

\begin{abstract}
In several research activities, the ab-initio calculations have become a vital tool for many research scientists (especially the physicists and the chemists). Pseudopotential plane wave method based on density functional perturbation theory within the Teter and Pade exchange-correlation functional form of the local density approximation is applied to study the anisotropy and pressure dependency of the mechanical properties of Boron-bismuth compound. The independent elastic compliance constants, the mechanical behavior, the phase transition, the volume collapse, the Young's modulus and the Poisson's ratio for directions within the important crystallographic planes under pressure are studied. The Debye temperature and the melting point are also predicted.
\end{abstract}

Keywords: PP-PW Method; Mechanical Properties of Semiconductors; Boron-Bismuth Compound.

\section{Introduction}

The mechanical properties such as: plasticity, hardness and brittleness of semiconductor materials are no less important than the thermoelectric and other physical properties of those materials to assure high reliability of respective products, instruments and devices [1]. The elastic constants of semiconductor materials provide a link between the mechanical and dynamical behavior of crystals, and give important information concerning the nature of the forces operating in solids [2]. In particular, they provide information on the brittleness and stiffness of materials, and their ab initio calculation requires precise methods [2]. So the mechanical properties of semiconductor materials play an important role on the fabrication of modern semiconductor instruments and devices. And because there are no data available in the literature of the effect of hydrostatic pressure on several of these quantities for boron bismuth (BBi) compound, we used density functional perturbation theory (DFPT) and as implemented in the ABINIT code to calculate series of mechanical constants and moduli under pressure of this compound.

The structural parameters and electronic behavior of boron-bismuth (BBi) in its zincblende phase have been studied by Madouri et al [3] using the full potential linearized augmented plane wave method. Under hydrostatic pressure the low pressure phase of material is destabilized and structural phase transition occurs. For BBi material, the phase transition appears at pressure of $31.74 \mathrm{GPa}$ [4]. Singh et al. [5] used the three-body interactions potential (TBIP) approach to study the elastic properties of this compound; they have also studied the phase transition pressure from zincblende (B3) phase to Rock-salt (B1) phase of BBi compound, and they found a value of pressure at about $34 \mathrm{GPa}$. In the present work, we report ab-initio calculations of the hydrostatic pressure effect on the elastic compliance constants and some others mechanical quantities of BBi compound in its structure (B3) phase using the pseudopotential plane wave method in the framework of the density functional perturbation theory [6], within the local density approximation (LDA). The mechanical stability criteria and the volume collapse are also studied.

\section{Computational methods}

Our calculations were performed by using the density functional perturbation theory and implemented in the ABINIT code [7-9]. ABINIT code is a package whose main program allows one to find the total energy, electronic structure and other properties such as elastic, optic, and dynamic of periodic solids within density functional theory, using 
pseudopotentials and a plane-wave basis-set. It is a common project of the Université Catholique de Louvain, Corning Incorporated, the Université de Liège, the Commissariat à l'Energie Atomique, Mitsubishi Chemical Corp., the Ecole Polytechnique Palaiseau and other contributors.

The pseudopotentials of Trouiller-Martins [10] in the context of the LDA are employed in the computation. The electron-electron interaction was considered within the LDA by Teter-Pade parameterization [11], which reproduces the correlation scheme of Perdew and Wang [12].

The two parameters that affect the accuracy of calculations are the kinetic energy cutoff which determines the number of plane-waves in the expansion and the number of special k-points used for the Brillouin zone (BZ) integration. The BZ integrations were replaced by discrete summations over a special set of k-points, using the standard k-point technique of Monkhorst and Pack [13] where the k-point mesh used is $(8 \times 8 \times 8)$. The plane-wave energy cut-off to expand the wave functions is set to be 90 Hartree. Careful convergence tests show that with these parameters, the relative energy is converged to better than $10^{-5}$ Hartree.

\section{Results and discussions}

\subsection{Elastic compliance constants}

The first goal of the present work is to report the pressure dependency of the elastic compliance constants for (B3) BBi material. In the case of the cubic crystals, the elastic compliance tensor $[\mathrm{S}]$ contains only three elastic compliance constants $\left(S_{11}, S_{12}\right.$ and $\left.S_{44}\right)$ [14]. The obtained values of the compliance constants $S_{11}, S_{12}$ and $S_{44}$ in $\left(10^{-2} \mathrm{GPa}^{-1}\right)$ of $(B 3)$ $\mathrm{BBi}$ at zero pressure are: $0.887,-0.231$ and 1.340 respectively. Unfortunately, there are no data available in the literature on the elastic compliance constants $S_{\mathrm{ij}}$ for this compound. Future experimental work or other theoretical calculations will contribute with more tests for the validating of our calculated results of these parameters.

The pressure variation of the elastic compliance constants $\mathrm{S}_{i j}$ of (B3) BBi material up to $48 \mathrm{GPa}$ is depicted in figure1. We observe that the constants $S_{11}, S_{12}$ and $S_{44}$ decrease gradually with the increasing of the hydrostatic pressure and with a negative sign for the constant $S_{12}$.

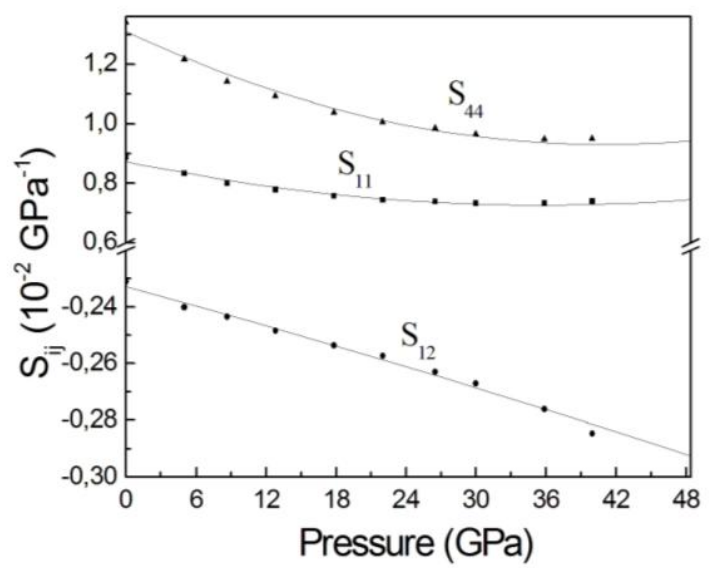

Fig. 1: Elastic Compliance Constants $S_{\mathrm{ij}}$ versus the Hydrostatic Pressure of the (B3) BBi.

\subsection{Young's modulus and Poisson's ratio}

The second goal of this work is to report the pressure dependency of the Young's modulus $\mathrm{Y}$ and Poisson's ratio $v$ for (B3) BBi compound. Young's modulus which is a measure of longitudinal elongation for isotropic solids, it is a very important mechanical coefficient in engineering of materials science; it is a quantity which is anisotropic for cubic zinc blende crystals (i.e.: strongly dependent with the directions) [14]. The Young's modulus $Y$ for an arbitrary crystallographic direction $\mathrm{x}$ can be given as a function of the elastic compliance constants $\mathrm{S}_{\mathrm{ij}}$ by the following expression [14]

$1 / Y=S_{11}-2\left(S_{11}-S_{12}-0.5 S_{44}\right)\left(x_{1}^{2} x_{2}^{2}+x_{2}^{2} x_{3}^{2}+x_{1}^{2} x_{3}^{2}\right)$

Where the $x_{i}$ values are the direction cosines for $x$.

The obtained values of the Young's modulus of BBi compound at different pressure $\mathrm{p}$ for the important directions are presented in figure 2. As shown on this figure, that all the curves increase with the increasing of the hydrostatic pressure. Three Analytical relations for the pressure dependency of (B3) Boron-bismuth Young's modulus Y are given by the following a quadratic fit, i.e.: 
$Y_{\{100\}<<001>}=112.93+1.547 \mathrm{p}-2.59 \times 10^{-2} \mathrm{p}^{2}$

$Y_{\{100\}<011>}=151.18+3.285 \mathrm{p}-4.4 \times 10^{-2} \mathrm{p}^{2}$

$Y_{\{10\} /<111>}=170.80+4.466 \mathrm{p}-5.21 \times 10^{-2} \mathrm{p}^{2}$

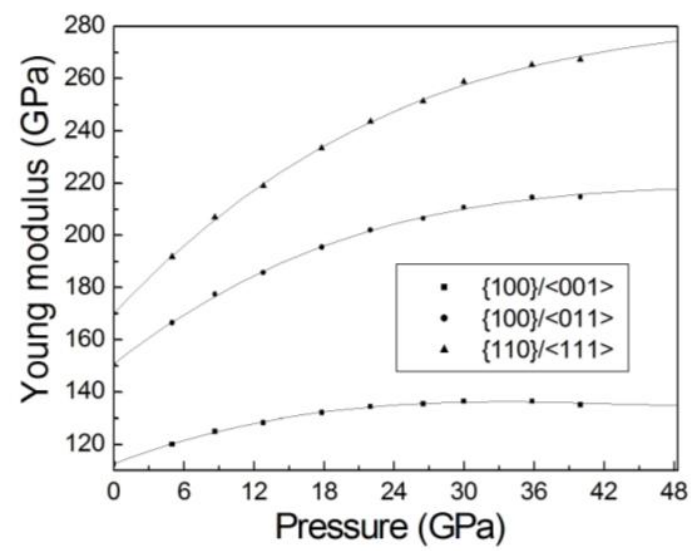

Fig. 2: Young's Modulus for Important Directions versus the Hydrostatic Pressure of (B3) BBi Compound.

Another important engineering coefficient in the fabrication of modern semiconductor devices is the Poisson's ratio $v$, it is also varied with the orientation in the cubic zinc blende crystals [14]. For isotropic solids, the Poisson's ratio $v$ is a measure of the transverse contraction [15]. If a longitudinal stress in the direction $\mathrm{x}$ and the transverse strain along the orthogonal direction y are under consideration, the Poisson's ratio $v$ can be given by [14].

$v=-\frac{\left[S_{12}+\left(S_{11}-S_{12}-0.5 S_{44}\right)\left(x_{1}^{2} y_{1}^{2}+x_{2}^{2} y_{2}^{2}+x_{3}^{2} y_{3}^{2}\right)\right]}{\left[S_{11}-2\left(S_{11}-S_{12}-0.5 S_{44}\right)\left(x_{1}^{2} x_{2}^{2}+x_{2}^{2} x_{3}^{2}+x_{1}^{2} x_{3}^{2}\right)\right]}$

The obtained values of $v$ at different pressure up to $48 \mathrm{GPa}$ for important crystallographic directions are presented in figure 3. As shown on this figure, that also all the curves (except for the Plane $\{100\}$ with direction $m=\langle 011\rangle$, and $n=$ $<0 \overline{1} 1>$ ) of the Poisson's ratio $v$ increase also gradually with the increasing of the hydrostatic pressure.

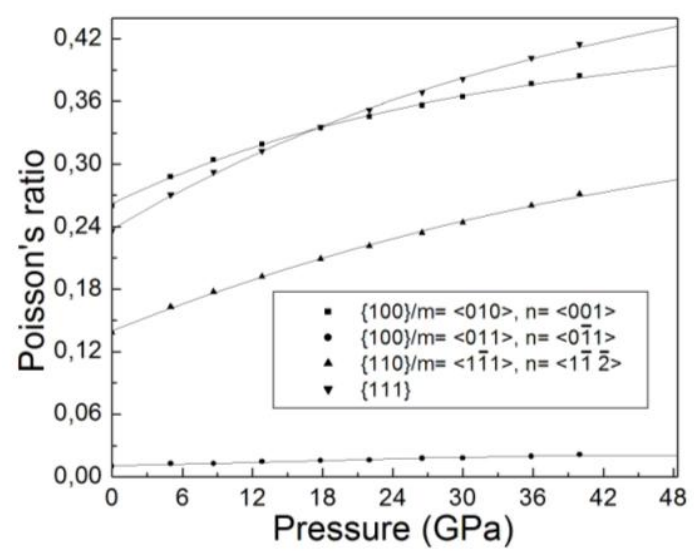

Fig. 3: Poisson's Ratio $v$ for Important Directions versus the Hydrostatic Pressure of (B3) BBi Compound.

Four Analytical relations for the pressure dependence of (B3) BBi Poisson's ratio $v$ are given by the following a quadratic fit, i.e.:

$$
\begin{aligned}
& v_{\{100\} / \mathrm{m}=\langle 010\rangle, \mathrm{n}=\langle 001\rangle}=0.262+5.27 \times 10^{-3} \mathrm{p}-6.34 \times 10^{-5} \mathrm{p}^{2} \\
& v_{\{100\} / \mathrm{m}=<011\rangle, \mathrm{n}=\langle 0 \overline{1} 1\rangle}=1.05 \times 10^{-2}+3.57 \times 10^{-4} \mathrm{p}-3.23 \times 10^{-6} \mathrm{p}^{2}
\end{aligned}
$$


$v_{\{100\} / \mathrm{m}=\langle 1 \overline{1} 0>, \mathrm{n}=\langle 1 \overline{12}\rangle}=0.140+4.61 \times 10^{-3} \mathrm{p}-3.86 \times 10^{-5} \mathrm{p}^{2}$

$v_{\{100\}}=0.237+6.74 \times 10^{-3} \mathrm{p}-6.64 \times 10^{-5} \mathrm{p}^{2}$

In the following section, we will discuss some mechanical properties as ductility and brittleness of our material. The ductility and brittleness of materials are of substantial importance and can be obtained usually from the Pugh's index of ductility $\mathrm{R}_{\mathrm{G} / \mathrm{B}}$ (called also the inverse ratio) or from the Poisson's ratio $v$ (for more detail on the study of ductility and brittleness of materials, please see the Ref. [16]). The effect of pressure on the inverse ratio $R_{\mathrm{G} / \mathrm{B}}$ is illustrated in Fig. 4 (a). Based on $\mathrm{R}_{\mathrm{G} / \mathrm{B}}$ quantity, for $\mathrm{BBi}$ compound, Fig. 4(a) shows that it is of brittle in nature from 0 to $24.7 \mathrm{GPa}$ and it is ductile in nature with an increase in pressure up to $48 \mathrm{GPa}$.

The pressure dependency of Poisson's ratio (here the average Poisson's ratio is taken) is also illustrated in Fig. 4 (b). Based on Poisson's ratio rule, two different values (0.26 and 0.33) [16] of the exact border between the two types of mechanical behavior have been mentioned. So, if the value of the Poisson's ratio is less than 0.26 (0.33), the material is considered brittle; otherwise (i.e. if $v$ is more than $0.26(0.33)$ ) the material behaves in a ductile manner [16]. For BBi compound, Fig. 4 (b) shows that it is of brittle in nature from 0 to $15 \mathrm{GPa}$ (0 to $42 \mathrm{GPa}$ ) and it is ductile in nature with the increasing in pressure up to $48 \mathrm{GPa}$.
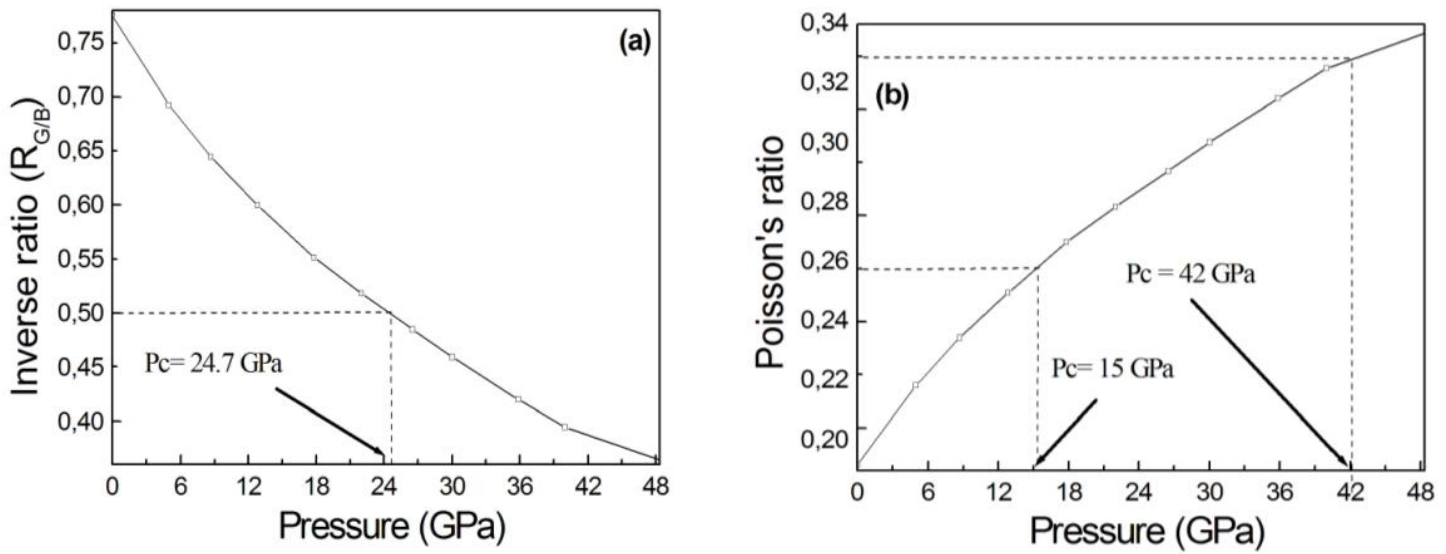

Fig. 4: Variation of Poisson's Ratio $v$ (a), and The Inverse Ratio $\mathrm{R}_{\mathrm{G} / \mathrm{B}}(\mathrm{b})$ of (B3) BBi Compound with Pressure.

Another quantity is also used to study the ductility and brittleness of materials; it is named the Cauchy's pressure. This quantity is defined as the difference between the elastic constants $\left(\mathrm{C}_{12}-\mathrm{C}_{44}\right)$. If the pressure is positive (i.e.: $\mathrm{C}_{12}-\mathrm{C}_{44}>$ 0 ), the material is expected to be ductile in nature, and in another case, i.e. if the pressure is negative $\left(C_{12}-C_{44}<0\right)$, the material is expected to be brittle [17].

\subsection{Mechanical stability criteria}

Usually the Gibbs-free energies (or the enthalpies at $\mathrm{T}=0 \mathrm{~K}$ ) are used to study the structural phase transition under pressure. But also the structural phase transition of crystal material can be investigated with the help of the elastic stability criteria. The structural stability under compressions of BBi compound was studied by using the generalized elastic stability criteria which are given by the following relations [18]:

$K=\frac{1}{3}\left(C_{11}+2 C_{12}+P\right)>0, G=\frac{1}{2}\left(C_{11}-C_{12}-2 P\right)>0$ and $G^{\prime}=C_{44}-P>0$

Where $\mathrm{C}_{\mathrm{ij}}$ are the elastic stiffness constants and $\mathrm{P}$ is the pressure

The elastic stiffness constants $C_{i j}$ and the elastic compliance constants $S_{i j}$ are related by the following expressions [14]:

$C_{11}=\left(S_{11}+S_{12}\right) /\left[\left(S_{11}-S_{12}\right)\left(S_{11}+2 S_{12}\right)\right], \quad C_{12}=\left(-S_{12}\right) /\left[\left(S_{11}-S_{12}\right)\left(S_{11}+2 S_{12}\right)\right], \quad$ and $\quad C_{44}=1 / S_{44}$

At zero pressure, the elastic stability criteria are satisfied, indicating that this compound is elastically stable. As pressure is applied to (B3) BBi compound, it gets transformed from the (B3) phase into the site-ordered $\mathrm{NaCl}$ (B1) phase. Three Analytical relations for the pressure dependency of the generalized elastic stability criteria are given by:

$K=78.09+3.65 \mathrm{p}-0.01 \mathrm{p}^{2}$ 


$$
\begin{aligned}
& G=45.15-6.75 \times 10^{-1} \mathrm{p}-5.42 \times 10^{-3} \mathrm{p}^{2} \\
& G^{\prime}=75.64+4.07 \times 10^{-1} \mathrm{p}-1.58 \times 10^{-2} \mathrm{p}^{2}
\end{aligned}
$$

We find that $\mathrm{G}$ decreases gradually with the pressure and vanishes at about $48 \mathrm{GPa}$; so the phase transition occurs at pressure of $48 \mathrm{GPa}$. This value is listed in table 1 and compared with the available theoretical data [4], [5], [19-21]. Our calculated value of the transition pressure is very higher than the previously calculated data [4], [5], [19-21].

\begin{tabular}{|c|c|c|c|c|c|}
\hline Parameter & Our work & Other works & & & \\
\hline $\mathrm{Pt}$ & 48 & $31.74[4]$ & 35.38[19]DM & $24.5[20]$ & $56.37[21]$ \\
\hline
\end{tabular}

Table 1: Transition Pressure $\mathrm{P}_{\mathrm{T}}$ (GPa) of (B3) BBi Material in Comparison with the Theoretical Data [4], [5], [19], [20] and [21].

The equation of state curves of BBi compound (plotted between $\mathrm{V}(\mathrm{P}) / \mathrm{V}(0)$ and the pressure) for both zinc blende (B3) and Rock-salt (B1) phases obtained in this work are plotted in figure 5. From this curves, we can estimate the volume collapse in the point of transition (in our case at $48 \mathrm{GPa}$ ).

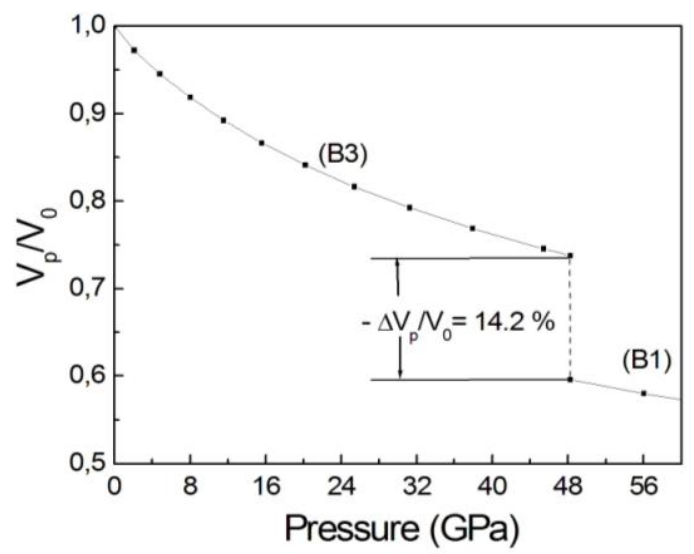

Fig. 5: Variations of the Ratio $\mathrm{V}_{\mathrm{P}} / \mathrm{V}_{0}$ of (B3) and (B1) BBi with Hydrostatic Pressure.

Our result of the volume collapse for (B3) BBi compound is given in table 2 and compared with the available theoretical data [4], [5]. Unfortunately, to the best of our knowledge, there are a little theoretical data [4], [5] available in the literature on this parameter for this compound.

Table 2: Volume Collapse of (B3) BBi Compound, in Comparison with the Available Theoretical Values [4], [5].

\begin{tabular}{llll}
\hline & Our work & Other works & $11.2[5]$ \\
\hline Volume collapse $(\%)$ & 14.2 & $6.6[4]$ & 11.2 \\
\hline
\end{tabular}

The Debye temperature is usually obtained by using the average sound velocity. But other formulas are largely used; among those formulas largely used for calculation of the Debye temperature of the cubic crystal materials is that proposed by Blackman [22]. It relating the Debye temperature and the elastic stiffness constants $\mathrm{C}_{\mathrm{II}}$; it is given as [22]:

$\theta_{D}^{3}=(3.15 / 8 \pi)(h / k)^{3}\left(n / g^{3 / 2} \mathrm{v}_{a}\right)\left(C_{11}-C_{12}\right)^{1 / 2}\left(C_{11}+C_{12}+2 C_{44}\right)^{1 / 2}\left(C_{44}\right)^{1 / 2}$

Where: $\mathrm{h}$ is the Planck's constant, $\mathrm{k}$ is the Boltzmann constant, $\mathrm{n}$ is the number of atoms in unit cell of volume $\mathrm{v}_{\mathrm{a}}$ and $\mathrm{g}$ is the crystal density. The elastic stiffness constants $C_{\mathrm{ij}}$ are related to the elastic compliance constants $\mathrm{S}_{\mathrm{IJ}}$ by the formula of Eq. (6). Our calculated values of $\theta_{\mathrm{D}}$ of $\mathrm{BBi}$ compound is listed in table 3, and compared with the available theoretical data [19-21], [23-25]. Our obtained value is in agreement with other theoretical results. Our obtained value (337.75 K) is in good agreement with the theoretical value $331.56 \mathrm{~K}$ cited in the Ref. [20]; the deviation being only about $1.87 \%$. For $\mathrm{A}^{\mathrm{III}}-\mathrm{B}^{\mathrm{V}}$ binary semiconductor compounds, a linear relationship between the Debye temperature and the melting point $T_{m}$ was proposed; it is given by the following formula [26]:

$$
\theta_{D}=-C_{1}+C_{2} T_{m}
$$

Where $C_{1}$ and $C_{2}$ are constants, the numerical values of the constants $C_{1}$ and $C_{2}$ are: $153.40 \mathrm{~K}$ and 0.354 respectively. Using the formula of Eq. (8), we can estimate approximately the value of $\mathrm{T}_{\mathrm{m}}$ from the following expression: 
$T_{m}=\left(\theta_{D}+C_{1}\right) / C_{2}$

The calculated $\mathrm{T}_{\mathrm{m}}$ of BBi material is about $1387.43 \mathrm{~K}$; it's listed in table 3, and compared with the available theoretical data [19], [20], [23-25]. Our obtained value of $T_{m}$ is in excellent agreement with other theoretical values, especially the value $1424 \pm 300 \mathrm{~K}$ cited in the Ref. [20] and the value $1312.866 \mathrm{~K}$ obtained by Ustundag et al. [24]; the deviations between our value and these cited in the Ref. [20] and in the Ref. [24] are only about $2.57 \%$ and $5.68 \%$ respectively.

Table 3: Debye Temperature and Melting Point f BBi Compound Along with Those Computed Through other Methods [19], [20], [21], [23], [24] and [25].

\begin{tabular}{|c|c|c|c|c|c|c|}
\hline Parameter & Our work & Other works & & & & \\
\hline$\theta_{\mathrm{D}}(\mathrm{K})$ & 337.75 & $\begin{array}{l}324.11[19] \\
370.23[25]\end{array}$ & $331.56[20]$ & $311.85[21]$ & $352.56[23]$ & 324.806 [24] \\
\hline $\mathrm{T}_{\mathrm{m}}(\mathrm{K})$ & 1387.43 & 1467 [19] & $1424[20]$ & $1522[23]$ & $1312.866[24]$ & $1479.18[25]$ \\
\hline
\end{tabular}

\section{Conclusion}

The effect of the hydrostatic pressure on the elastic compliance constants of BBi material are obtained, we found that these elastic compliance constants decrease almost quadratic with the increasing of hydrostatic pressure (with a negative sign for the constant $S_{12}$ ). The obtained values of the elastic compliance constants $S_{i j}$ were used to predict the orientation dependency of two mechanical quantities: Young's modulus Y, and Poisson's ratio $v$.

The value of pressure separates the ductile and brittle nature is also obtained; at zero pressure the BBi compound is of brittle in nature. In the investigation of the stability criteria, the results showed a phase transition pressure from the zinc blende to Rock-salt phase at around $48 \mathrm{GPa}$. The volume collapse, the Debye temperature and the melting point are also predicted. The percentage deviations of $1.87 \%$ and $2.57 \%$ were obtained in the cases of Debye temperature and melting point respectively.

\section{References}

[1] V. P. Mykhalchenko, "Correlation between mechanical properties of diamond-like semiconductor compounds and lattice thermal conductivity", Journal of Thermoelectricity Vol.4, (2012), pp. 5-13. http://jt.inst.cv.ua/jt_04_2012_eng.pdf.

[2] H. Koc, A. Yildirim \& E. Deligöz, "Ab initio calculations of the elastic, electronic, optical, and vibrational properties of PdGa compound under pressure", Chinese Physics B, Vol. 21, No. 9, (2012), pp. 097102 (8 pages). http://iopscience.iop.org/1674-1056/21/9/097102/.

[3] D. Madouri \& M. Ferhat, "How do electronic properties of conventional III-V semiconductors hold for the III-V boron bismuth BBi compound?" Physica Status Solidi (b), Vol.242, No.14, (2005), pp. 2856-2863, http://dx.doi.org/10.1002/pssb.200441121.

[4] E. Deligöz, Bazi İkili (CdS, CdSe, CdTe, CdF2, AlBi, BBi) bilesiklerin yapisal, elektronik, elastik, termodinamik ve titresimsel özelliklerinin ab initio yöntemle incelenmesi, Thesis of Doctorate, GAZI University, ANKARA (2007). http://fbetezbankasi.gazi.edu.tr/pdf-indir/22200992.

[5] S. Singh, \& M. Sarwan, "Structural phase transition and high pressure elastic behavior of BX $(\mathrm{X}=\mathrm{Sb}, \mathrm{Bi})$ compounds", Journal of optoelectronics and advanced materials, Vol.12, No.10, (2010), pp. 2106-2112. http://www.joam.inoe.ro/download.php?idu=2623.

[6] S. Baroni, P. Giannozzi, and A. Testa, "Green's Function approach to linear response in solids" Physical Review Letters, Vol. 58, No.18, (1987), pp. 1861-1864. http://www.ncbi.nlm.nih.gov/pubmed/10034557. http://dx.doi.org/10.1103/PhysRevLett.58.1861.

[7] X Gonze, J.-M. Beuken a, R. Caracas, et al, "First-principles computation of material properties: the ABINIT software project", Computational Materials Science, Vol. 25, (2002), pp. 478-492. www.researchgate.net/computationmaterial/9fcfd50c5f4ec3f833.pdf.

[8] X. Gonze, G. M. Rignanese, M. Verstraete, et al. "A brief introduction to the ABINIT software package", Zeitschrift für Kristallographie, Vol. 220, No.5-6, (2005), pp. 558-562. dx.doi.org/10.1524/zkri.220.5.558.65066.

[9] S. Goedecker, "Fast Radix 2, 3, 4, and 5 kernels for fast Fourier transformations on computers with overlapping multiply-add instructions", SIAM Journal on Scientific Computing, Vol. 18, No.6, (1997), pp. 1605-1611. https://comphys.unibas.ch/PUBLICATIONS/FFTker.pd. http://dx.doi.org/10.1137/S1064827595281940.

[10] N. Troullier and J. L. Martins, "Efficient pseudopotentials for plane-wave calculations", Physical Review B, Vol. 43, No. 3, (1991), pp. 19932006. http://inside.mines.edu/ Zhiwu/research/papers/B04_tm.pdf http://dx.doi.org/10.1103/PhysRevB.43.1993.

[11] S. Goedecker, M. Teter \& J. Hutter, "Separable dual-space gaussian pseudopotentials", Physical Review B, Vol.54, No.3, (1996), pp. 17031710. http://dx.doi.org/10.1103/PhysRevB.54.1703.

[12] J. P. Perdew \& Y. Wang, "Accurate and simple analytic representation of the electron-gas correlation energy", Physical Review B, Vol. 45, No. 23, (1992), pp. 13244-13249. link.aps.org/doi/10.1103/PhysRevB.45.13244. http://dx.doi.org/10.1103/PhysRevB.45.13244.

[13] H .J. Monkhorst \& J. D. Pack, "Special points for Brillouin-zone integrations", Physical Review B, Vol.13, No.12, (1976), pp. 5188-5192. http://dx.doi.org/10.1103/PhysRevB.13.5188.

[14] S. Adachi, "Properties of Group-IV, III-V and II-VI Semiconductors", John Wiley \& Sons, England, (2005). ISBN 0-470-09032-4. http://dx.doi.org/10.1002/0470090340.

[15] R. E. Newnham, "Properties of materials: Anisotropy, Symmetry, Structure", Oxford University Press, USA, (2005). ISBN 0-19-852075-1.

[16] D. Varshney, G. Joshi, M. Varshney \& S. Shriya, "Pressure induced mechanical properties of boron based pnictides", Solid State Sciences, Vol. 12, (2010), pp. 864-872. DOI:10.1016/j.solidstatesciences.2010.02.003. http://dx.doi.org/10.1016/j.solidstatesciences.2010.02.003.

[17] M. Shoaib, G. Murtaza, R. Khenata, M. Farooq, \& Roshan Ali, "Structural, elastic, electronic and chemical bonding properties of $A B(A=S c$, $\mathrm{Y}, \mathrm{La} ; \mathrm{B}=\mathrm{N}, \mathrm{P}$, As, Sb, Bi) from first principles", Computational Materials Science, Vol.79, (2013), pp. $239-247$. http://dx.doi.org/10.1016/j.commatsci.2013.06.015.

[18] S. Daoud, N. Bioud, \& N. Bouarissa, "Structural phase transition, elastic and thermal properties of boron arsenide: Pressure-induced effects", Materials Science in Semiconductor Processing, Vol. 31, (2015), pp. 124- 130. http://dx.doi.org/10.1016/j.mssp.2014.11.024.

[19] K. Amara, B. Soudini, D. Rached \& A. Boudali, "Molecular dynamics study on thermomechanical properties of cubic BBi", Computational Materials Science, Vol. 44, No. 2, (2008) pp. 635-640. DOI: 10.1016/j.commatsci.2008.04.023. http://dx.doi.org/10.1016/j.commatsci.2008.04.023. 
[20] S. Cui, W. Feng, H. Hu, Z. Feng \& Y. Wang, "First principles studies of phase stability, electronic and elastic properties in BBI compound", Computational Materials Science, Vol.47, No.4, (2010), pp. 968-972. http://www.sciencedirect.com/science/article/pii/S0927025609004418. http://dx.doi.org/10.1016/j.commatsci.2009.11.030.

[21] S. Daoud, N. Bioud \& N. Lebgaa, " Elastic and piezoelectric properties, sound velocity and Debye temperature of (B3) BBI compound under pressure", Pramana journal of physics, Vol. 81, No. 5, (2013), pp. 885-892. www.ias.ac.in/pramana/v81/p885/fulltext.pdf.

[22] M. Blackman, "On the calculation of characteristic temperatures from the elastic constants", Philosophical Magazine, Vol.42, No. 335, (1951), pp. 1441-1442. http://dx.doi.org/10.1080/14786445108560963.

[23] E. Deligöz, K. Colakoglu, Y. O. Ciftci, \& H. Ozisik, "The first principles study on boron bismuth compound", Computational Materials Science, Vol.39, No.3, (2007), pp. 533-540. http://www.sciencedirect.com/science/article/pii/S0927025606002321. http://dx.doi.org/10.1016/j.commatsci.2006.08.004.

[24] M. Ustundag, M. Aslan, \& Battal G. Yalcin, "The first-principles study on physical properties and phase stability of Boron-V (BN, BP, BAs, $\mathrm{BSb}$ and BBi) compounds", Computational Materials Science, Vol. 81, (2014) pp. 471-477. http://dx.doi.org/10.1016/j.commatsci.2013. 08.056 .

[25] S. Daoud, N. Bioud, N. Lebgaa \& R. Mezouar "Optoelectronic and thermal properties of boron- bismuth compound", International Journal of Physical Research", Vol. 2, No. 2, (2014), pp. 27-31. http://www.sciencepubco.com/index.php/IJPR/article/view/2760. http://dx.doi.org/10.14419/ijpr.v2i2.2760.

[26] V. Kumar, V. Jha, \& A. K. Shrivastava, "Debye temperature and melting point of II-VI and III-V semiconductors", Crystal Research and Technology, Vol. 45, No. 9, (2010) pp. 920-924. http://www.onlinelibrary.wiley.com/doi/10.1002/crat.201000268/pdf. http://dx.doi.org/10.1002/crat.201000268. 